\title{
«Acoma», numeri 1-5 (1971-1973), rivista diretta da Édouard Glissant
}

\section{Alessandro Corio}

\section{(2) OpenEdition}

1 Journals

\section{Edizione digitale}

URL: http://journals.openedition.org/studifrancesi/28123

DOI: 10.4000/studifrancesi.28123

ISSN: 2421-5856

\section{Editore}

Rosenberg \& Sellier

\section{Edizione cartacea}

Data di pubblicazione: 31 décembre 2006

Paginazione: 642-644

ISSN: 0039-2944

\section{Notizia bibliografica digitale}

Alessandro Corio, « «Acoma», numeri 1-5 (1971-1973), rivista diretta da Édouard Glissant », Studi

Francesi [Online], 150 (L | III) | 2006, online dal 30 novembre 2015, consultato il 08 novembre 2020. URL : http://journals.openedition.org/studifrancesi/28123 ; DOI : https://doi.org/10.4000/

studifrancesi.28123

\section{Questo documento è stato generato automaticamente il 8 novembre 2020}

\section{(c) $(1) \odot$}

Studi Francesi è distribuita con Licenza Creative Commons Attribuzione - Non commerciale - Non opere derivate 4.0 Internazionale. 


\title{
«Acoma», numeri 1-5 (1971-1973), rivista diretta da Édouard Glissant
}

\author{
Alessandro Corio
}

\section{NOTIZIA}

«Acoma», numeri 1-5 (1971-1973), rivista diretta da Édouard Glissant, Perpignan, Presses Universitaires de Perpignan, 2005, pp. 656.

1 La riedizione dei cinque numeri della rivista martinicana "Acoma», pubblicati in Martinica ed in Francia, tra il 1971 e il 1973, dalla casa editrice Maspero di Parigi (la quale ha poi interrotto la pubblicazione per motivi economici) rappresenta senz'altro un evento prezioso per tutti gli studiosi delle culture e delle letterature caraibiche e, più in generale, per quanti si occupino, ad un livello più o meno specialistico, di tematiche inerenti al colonialismo, al razzismo, alle culture della black diaspora e alle politiche anti- e post-coloniali. La rivista fu la prima di questo genere in Martinica e fu caratterizzata, fin dal progetto, da un approccio pluridisciplinare, aperto alle arti visive ed accogliente nei confronti di scrittori ed artisti delle Americhe come delle Antille. Il progetto nacque da un gruppo di studiosi, intellettuali e scrittori martinicani, diretti da Édouard Glissant, il quale nel 1967, due anni dopo il suo ritorno nell'isola natale dal "confino" politico a Parigi, aveva creato l'Institut Martiniquais d'Etudes (I.M.E.) allo scopo di combattere l'alienazione culturale di un paese a maggioranza nera, il cui sistema scolastico, diventato una vera e propria istituzione di assimilazione, era basato interamente sulla cultura franco-metropolitana. La rivista nasce appunto come luogo di pubblicazione dei lavori di ricerca dell'Istituto e dall'esigenza "politica", profondamente sentita come necessaria, di unire indissolubilmente il progetto di ricerca al campo di studio, per scavare a fondo la natura delle cause che hanno reso la società antillese una "società bloccata" a livello sociale, storico e psicologico e per aprire la strada a nuove possibilità di discorso e di azione che attivino processi reali di cambiamento. L'epigrafe, posta immediatamente sotto il titolo della rivista, ne enuncia chiaramente, per mezzo di una metafora vegetale, l'ambizione e l'ispirazione profonda: 
«L'acoma franc est un de plus hauts arbres du pays ... On remarque que fort longtemps après estre coupé, le cœur en est aussi sain, humide et plein de sève, que si on le venait de mettre par terre» (J.-B. du Tetre - Histoire naturelle des Antilles).

Dopo la lunga stagione della négritude, dopo la "loi de départimentalisation" del 1946 voluta da Aimé Césaire e dopo lo scioglimento nel 1961, su decreto del generale De Gaulle, del Front Antillo-Guyanais per la liberazione del popolo antillese, fondato da Glissant e dal suo amico Albert Béville (di cui uno degli obiettivi principali era l'integrazione delle Antille francesi nella regione caraibica, ritenuta una tappa essenziale per l'emancipazione culturale e politica), la situazione sociale, economica e politica in Martinica sembra giunta ad un punto di completo stallo, che si riassume drammaticamente nelle parole dello stesso GLISSANT: «Il faut supposer que la colonisation française en Martinique risque bientôt de parvenir au "stade suprême" de toute colonisation, qui est de dépersonnaliser complètement une communauté, de l'“absorber" dans un corpus extérieur, et qu'en ce sens la colonisation de la Martinique se révélerait alors comme l'une des rares colonisations "réussies" de l'histoire moderne» (p. 90).

3 Il breve periodo di pubblicazione della rivista $\mathrm{e}$, più in generale, tutti gli anni settanta si presentano oltremodo problematici per la riproposizione di una progettualità politica che tenga conto dei successi, ma anche dei drammatici fallimenti ed involuzioni dei processi di decolonizzazione reale avviati dopo la seconda guerra mondiale, trovandosi inoltre in un contesto, come quello dei Départements d'Outre-Mer francesi, dove un processo di presa di coscienza e di decolonizzazione non è mai stato avviato, essendo stato soffocato sul nascere dall'ideologia assimilazionista e dal mito della citoyenneté française. L'ideologia della négritude, con la sua proposta di un ritorno ad un'identità africana ancestrale, comincia a presentare elementi sospetti di essenzialismo, culturalismo e di alienazione e viene sentita come "allogena" ed "eteronoma" rispetto al contesto storicamente composito, meticcio e creolo dei carabi, nonché l'espressione ideologica della piccola borghesia di colore, subalterna nei confronti del grande capitale metropolitano (si veda l'ottima analisi di André LUCRÈCE, Le mouvement martiniquais de la négritude. Essai d'analyse d'un discours idéologique, pp. 93-123). Si rende invece sempre più necessaria un'attenzione ai problemi specifici del contesto antillese, la cui identità, che più tardi Glissant definirà "rizomatica", nasce da una violenta rottura, operata dalla tratta e dal sistema della schiavitù nelle piantagioni, con le "radici africane". Come la linfa che sopravvive a lungo nel tronco reciso dell'acoma, è proprio dalle molteplici storie e dalle tracce che si sono sedimentate, per quanto nascoste ed alienate, nelle profondità delle società antillesi che bisogna ripartire per ridare corpo ad una coscienza di popolo, che Glissant definisce "antillanité", per superare la frattura, che continua invece ad estendersi in modo sempre più disastroso accentuando il disequilibrio generale, tra la società e il proprio entour geografico, tecnologico e culturale.

Il primo numero della rivista è introdotto da una vibrante Lettre de Fort-de-France (pp. 10-32) di Jaques FoRman (all'epoca ministro degli Affari esteri del Black Power), un militante nero americano alla ricerca delle motivazioni profonde della sua lotta sulle tracce biografiche di Franz Fanon. La lettera mette in rilievo, quasi un manifesto suigeneris, l'ideale transculturale di lotta politica antirazzista, anticolonialista ed anticapitalista che muove questo gruppo di ricercatori, ben attenti a mantenere le distanze da qualsiasi tentazione ideologica ed elitaria e ben consapevoli che il loro 
approfondimento teorico ed intellettuale non potrà essere che uno "strumento" nelle mani di chi cercherà di tradurlo in azione e in cambiamento.

5 I quattro saggi raccolti nel primo numero sotto il titolo Introduction à quelques problèmes antillais, curati dal Gruppo di Ricerca dell'I.M.E. (Édouard Glissant, Michel Giraud, Georges Gaudi, Marlène Hospice, Roland Suvélor ecc.), si propongono come obiettivo proprio quello di dare avvio ad un serio lavoro di ricerca in scienze umane (sociologiche, etnografiche, economiche ecc.) condotto da studiosi delle Antille. La ricerca, servendosi degli strumenti del materialismo dialettico, della psicoanalisi, dell'analisi del discorso sociale ed ideologico in relazione alle tensioni ed alle dinamiche sociali, intende «élucider [...] la nature des barrages inhérents à la structure de la société antillaise et à la psyché générale de l'Antillais, héritages d'une longue histoire coloniale, qui freinent le développement d'une recherche antillaise autonome» (p. 34). Questo allo scopo di stabilire una piattaforma teorica comune per un lavoro di inchiesta sul campo che cerchi di unire teoria e pratica effettiva. Possiamo qui solamente accennare a due importanti saggi di Édouard GLISSANT, che saranno poi rielaborati ne Le Discours antillais (1981) e che pongono le basi di tutto il suo lavoro a venire: Structures et tensions de groupe en Martinique (pp. 35-47), che individua le cause del disequilibrio generale della collettività martinicana nella mancanza di una relazione equilibrata ed attiva col proprio spazio (a causa della doppia alienazione prodotta dai miti del ritorno all'Africa e da quello della cittadinanza francese o francisation) e col proprio tempo (a causa dell'assenza di una dimensione storica e di una memoria collettiva che possano guidare una proiezione progettuale verso l'avvenire); Introduction à une étude des fondements socio-historiques du déséquilibre mental (pp. 82-97), spinge ancora oltre l'analisi applicando una sorta di "psicoanalisi globale" o collettiva per individuare le cause "strutturali" della "morbosità generale" della collettività, scaturite dalla rimozione del proprio vissuto storico traumatico. Esse sono da identificarsi, secondo Glissant, proprio nella non-autonomia del circuito economico martinicano, che ha generato una distorsione a livello sociale tra funzione reale e funzione opinata dei gruppi sociali, impedendo così il nascere di conflitti sociali autonomi e "lotte di classe" che avrebbero reso possibile l'autonomia del paese rispetto alla madre-patria. Anche il saggio di Michel GIRAUD, Les conflits raciaux considérés comme substitut de la lutte des classes aux Antilles (pp. 48-61), applica l'analisi teorica del materialismo dialettico al razzismo, individuandone la funzione sociale legata allo sfruttamento economico dei gruppi subalterni, dalla sua genesi alle metamorfosi più recenti.

6 L'analisi teorica non è l'unica componente della rivista. Ad essa si affiancano testi di altra natura: innanzitutto testi letterari, teatrali come L'ombre de la falaise, oratorio di Henri CORBIN (pp. 98-114), poetici come La roche écrite (pp. 232-238) e Poème (549-557) di Arlet JOUANAKARÉA, Billons e Bois des Hauts di Édouard GLISSANT (pp. 295-318), Une affaire réglée di Jean MÉTELLus (pp. 558-561), Province ténébreuse di Henri CORBIN (pp. 323-326) e un estratto di Discours didactique dell'importante poeta quebecchese Gaston MIRON (pp. 447-462). Nel secondo numero della rivista viene presentata una nuova versione del racconto popolare martinicano Conte de Yé, raccolto da Lafcadio HEARN, seguito da un'interpretazione di Roland sURVELOR (pp. 327-358). Viene in seguito presentato il primo lavoro del gruppo di teatro dell'I.M.E., Histoire de nègre (pp. 359-400). Sono presenti inoltre analisi di carattere storico-politico su Haiti, come L'occupation américaine en Haiti di Suzy Castor e Le phénomène Duvallier di Rémy Ansèlme, e saggi di carattere più strettamente letterario come Littérature nègre aux U.S.A. di Marlène 
Hospice (pp. 115-131 e 217-231), Théâtre, conscience du peuple di Édouard Glissant (pp. 187-205) e L'image des États-Unis dans les cuvres des auteurs noirs de langue française di Juris Siléniks (pp. 618-627).

7 Non possiamo non citare le accurate Bibliografie critiche, presenti in ogni volume della rivista, e soprattutto lo spazio non indifferente dedicato all'arte figurativa di artisti delle Antille e delle Americhe, tra cui Poétique de la révolution chilienne (texte et dessins) di MATTA (pp. 153-165), Présentation de Camacho (pp. 319-322) e Présentation de Ferrer (pp. 592-596), entrambi artisti cubani. Non mancano infine, elemento fondamentale della rivista, interventi di più immediato impegno e militanza politica, come i testi intitolati Evénement che aprono i vari numeri, tra i quali l'esplicita denuncia, attraverso una Motion des enseignants de l'Institut Martiniquais d'Etudes (p.150), delle rappresaglie contro il movimento studentesco martinicano del 1971 e dell'assassinio senza motivo di un giovane studente a Fort-de-France da parte delle forze dell'ordine; infine il testo che chiude questo ricchissimo volume, Le dossier brésilien (pp. 648-650), una denuncia della repressione, delle torture e degli assassini in Brasile, durante il periodo della dittatura militare sostenuta dalla CIA. 